\title{
Cell-Free Therapies: Novel Approaches for COVID-19
}

\author{
Tatiana Maron-Gutierrez ${ }^{1,2 \star}$ and Patricia R. M. Rocco ${ }^{3,4,5}$ \\ ${ }^{1}$ Laboratory of Immunopharmacology, Oswaldo Cruz Institute, Oswaldo Cruz Foundation, Fiocruz, Rio de Janeiro, Brazil, \\ ${ }^{2}$ National Institute of Science and Technology for Neuroimmunomodulation, Rio de Janeiro, Brazil, ${ }^{3}$ Laboratory of Pulmonary \\ Investigation, Carlos Chagas Filho Institute of Biophysics, Federal University of Rio de Janeiro, Rio de Janeiro, Brazil, \\ ${ }^{4}$ National Institute of Science and Technology for Regenerative Medicine, Rio de Janeiro, Brazil, ${ }^{5}$ COVID-19 Virus Network, \\ Ministry of Science and Technology, and Innovation, Rio de Janeiro, Brazil
}

Keywords: COVID-19, SARS-CoV-2, cell-free therapies, extracellular vesicle (EV), MSC secretome, mitochondria

\section{INTRODUCTION}

First described in late 2019, coronavirus disease (COVID)-19, caused by the severe acute respiratory syndrome coronavirus 2 (SARS-CoV-2), rapidly escalated into a global pandemic with a high case fatality rate. COVID-19 patients with acute respiratory failure exhibit some distinctive pathological characteristics, which to some extent resemble the acute respiratory distress syndrome (ARDS), SARS, and Middle East respiratory syndrome (MERS); these include hypoxemia, diffuse alveolar damage with cellular exudates, extensive pulmonary inflammation, lung edema, and hyaline membrane formation (1). In addition to respiratory failure, these patients present with a dysfunctional systemic host response that affects multiple organs, including the central nervous, cardiovascular, renal, and gastrointestinal systems (2-4), as well as a wide range of coagulation disturbances, such as thrombocytopenia, sustained systemic clotting activation, massive thrombin and fibrin formation, and disseminated intravascular coagulation (5).

University of Hull, United Kingdom

Reviewed by:

Muhammad Nawaz,

University of Gothenburg, Sweden

Sally Yunsun Kim,

Imperial College London,

United Kingdom

*Correspondence:

Tatiana Maron-Gutierrez

tati.maron@gmail.com;

tatiana.maron@ioc.fiocruz.br

Specialty section:

This article was submitted to Inflammation,

a section of the journal

Frontiers in Immunology

Received: 13 July 2020

Accepted: 18 August 2020

Published: 18 September 2020

Citation:

Maron-Gutierrez T and Rocco PRM

(2020) Cell-Free Therapies: Novel

Approaches for COVID-19.

Front. Immunol. 11:583017.

doi: 10.3389/fimmu.2020.583017

\section{CURRENT AVAILABLE TREATMENTS AGAINST COVID-19}

Few therapies are effective for COVID-19 patients, and to date there are still no vaccines available. There are vaccine candidates in development and ongoing clinical trials, but they are expected to be available, at best, in early 2021 (6). Thus, novel effective and safe therapies are urgently required to treat COVID-19 patients (7). In this context, several clinical trials have begun pursuing new therapies as well as repurposing existing ones, largely through drug repositioning, including of antiviral, antimalarial, and anti-inflammatory agents. These therapies aim to target entry of the virus into host cells, multiplication of the viral genetic material, and/or the immune response and inflammatory process (6). Although some therapies shows promising results, they raise some concerns, such as limited cohort sizes, non-randomized trials, lack of considerations for gender, comorbidities, concurrent treatments, and route of drug delivery, among others (6). Early in the COVID-19 pandemic, corticosteroids were not recommended because of the adverse effects previously observed in influenza, SARS-CoV, and MERS-CoV infections (8). Nevertheless, a recent controlled, open-label trial of dexamethasone for up to 10 days resulted in lower 28-day mortality in mechanically ventilated patients (9).

Mesenchymal stromal cell (MSC)-based treatment has been proposed as a suitable therapeutic approach for COVID-19 (10). As MSCs have the potential to interact directly with immune cells, their transplantation may improve outcomes in COVID-19 patients through modulation of the immune response, mitigation of the inflammatory cascade, and promotion of tissue repair and regeneration $(11,12)$. In addition, CD147, the second entry receptor for SARS-CoV-2, can be expressed by tissue-specific stem cells (13). Together with the loss of airway epithelial cells by 
viral infection and replication, the additional loss of regenerating stem cells may be responsible for diminished cellular and lung regeneration (13). Cell-based therapies have been quite extensively studied for potential applicability in COVID-19, especially given the short time since the onset of the pandemic $(14,15)$; however, due to the risk of macro- and microthrombosis, cell-free therapies may be more appealing. Cell-free therapies might decrease injury to different organs, such as lung, heart, kidney, liver, and brain, as well as reduce thrombus formation and endothelial inflammation (Figure 1).

\section{CELL-FREE THERAPIES FOR COVID-19}

Cell-free therapies, such as the MSC secretome (obtained as conditioned medium) and extracellular vesicles (EVs) from MSCs, have been studied in ARDS (16) and multiple organ dysfunction syndrome (MODS) $(17,18)$ for their antiinflammatory and anti-fibrogenic effects, as well as their epithelial and endothelial regenerative properties. However, many researchers and international societies, including the International Society for Extracellular Vesicles (ISEV) and the International Society for Cellular and Gene Therapies (ISCT), have expressed concern regarding the use of EVs-whether derived from MSCs or from other cell sources-in the treatment of COVID-19 (19). Clinical trials are encouraged; however, the use of EVs for any purpose in COVID-19 is not endorsed by ISEV and ISCT until proper regulation of manufacturing, quality control protocols, and clinical trial design are in place, in order to avoid the stem-cell industry trying to sell unregulated MSC treatments (19). In this context, the implementation of computer-controlled bioreactors (20) and the development of standard operating procedures (SOPs) for obtaining a Good Manufacturing Practice (GMP)-grade MSC secretome and its components are necessary for clinical applications (21). These must be reproducible, scalable, and well-controlled to limit heterogeneity and enhance predictability in the composition and function of secretome-derived products (22). Further studies are still needed to better understand the best route of cell-free therapy

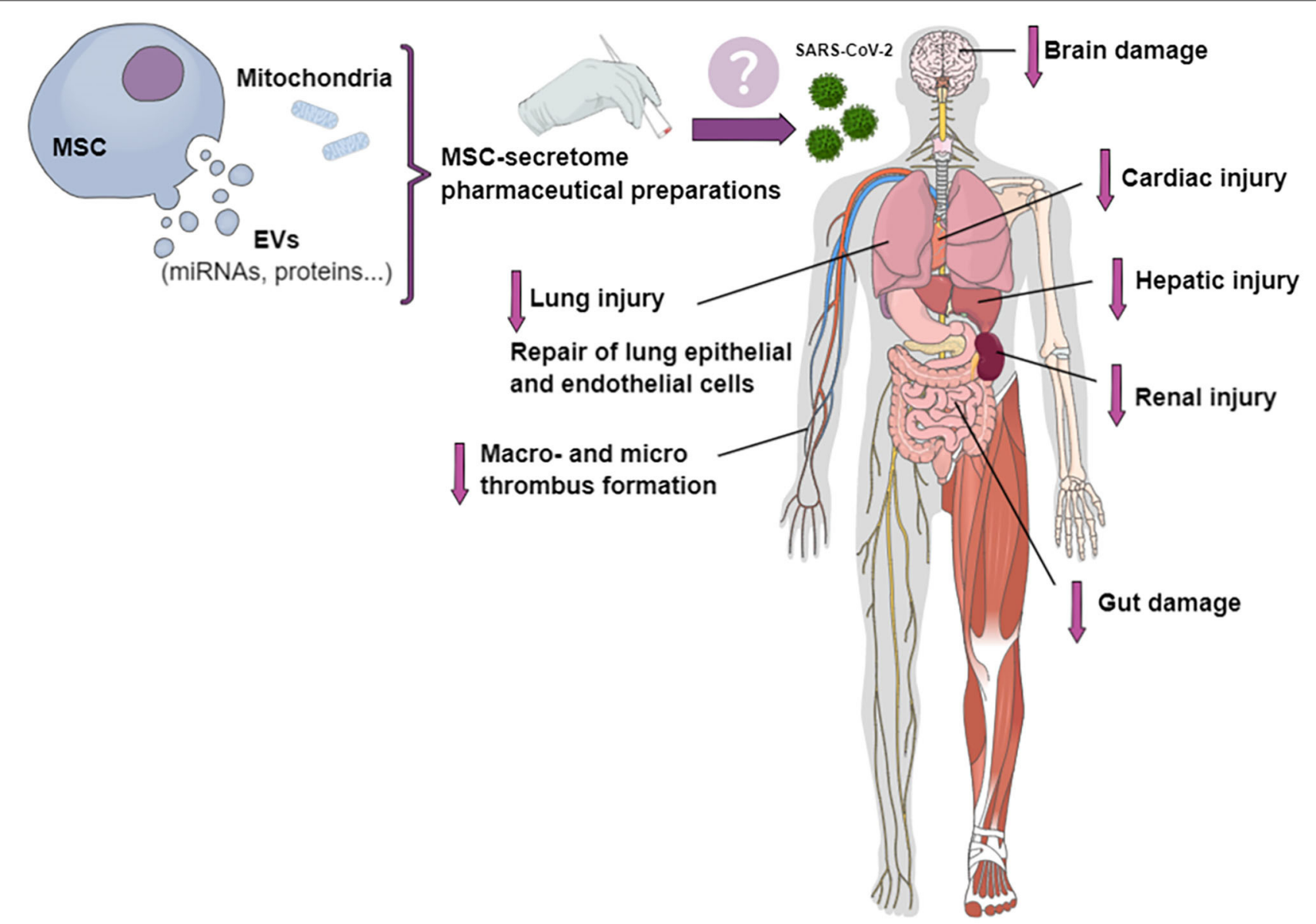

FIGURE 1 | Potential effects of pharmaceutical preparations of MSC secretome on different organs involved in COVID-19. The MSC secretome, in the form of conditioned medium containing extracellular vesicles (EVs) and mitochondria, could be transformed into a stable product for the treatment of patients with COVID-19. Secretome-based therapies might mitigate cardiac, kidney, liver, nervous system, and lung injury; decrease macro- and micro-thrombus formation and endothelial inflammation; and repair lung epithelial and endothelial cells. 
delivery, dose, and timing of administration (23). Moreover, important factors should be taken into consideration such as the culture medium, cell and tissue source, donor variability, and culture conditions (cell priming with hypoxia, biochemical or mechanical stimuli, three-dimensional spheroid culture, among others), as well as the timing and method of MSC-secretome harvesting $(20,22)$. In short, cell-free therapies could be a more suitable treatment for COVID-19 than MSCs, but additional investigations are required (2).

\section{THERAPEUTIC STRATEGIES INVOLVING THE MSC SECRETOME IN COVID-19}

The MSC secretome is a complex mixture of soluble components (growth factors and cytokines), a vesicular portion that comprises EVs, and cell organelles (e.g., mitochondria) (2426). Considering that SARS-CoV-2 infection is being associated with an increased inflammatory process (27), we believe that MSC secretome products might help reverse COVID-19related immune dysregulation, due to their anti-inflammatory, immunomodulatory, and regenerative effects (14). The MSC secretome has properties similar to those of its parent MSCs (28). Moreover, the secretome is generally considered safer than parent cells, since it (1) lacks the potential for endogenous tumor formation, as it cannot self-replicate; (2) can be classified as non-immunogenic, due to the limited number of antigenic components; and (3) may lead to less formation of emboli when injected intravenously $(14,29)$. As recently reported elsewhere, the MSC secretome (in the form of conditioned medium) can be stored more easily than MSCs (14), which is an important consideration given the lack of adequate facilities in developing countries. Transforming MSC-secretome components into a freeze-dried, stable powder product which can be reconstituted for intravenous injection or inhalation might be a suitable approach for the treatment of patients with COVID-19 (Figure 1) (17).

\section{MITOCHONDRIA FOR RESTORING BIOENERGETICS AND MITIGATING INFLAMMATION}

Mitochondria are intracellular organelles that play a vital role in cellular homeostasis and enable stress adaptation (30). Most cellular energy generation takes place in the mitochondria (31), and excessive mitochondrial dysfunction leading to defects in energy flow leads to unsustainable maintenance of life and adaptation to stress (30). One of the main mechanisms associated with the pathophysiology of sepsis is mitochondrial dysfunction $(32,33)$. In 2012, the first evidence that MSCs restore alveolar bioenergetics through $\mathrm{Cx} 43$-dependent alveolar attachment and mitochondrial transfer was observed in experimental ARDS (25). In 2015, Phinney et al. observed mitochondrial transfer from MSCs to macrophages in response to oxidative stress (34). Recently, Court et al. investigated the effect of MSCmediated transfer of mitochondria on lymphoid cells. They observed mitochondria-labeled MSCs mainly in CD4+T cells, paving the way for exploration of organelle-based therapies in immune diseases (26). Interestingly, MSCs are not the only cell type able to transfer mitochondria. Lipopolysaccharide (LPS)stimulated monocytes release free and microvesicle-associated mitochondria as part of their secretome (35). These studies demonstrate the complexity of cell-to-cell communication by identifying mitochondria as a source for target cells to restore their bioenergetics, enable immunomodulatory effects, and suppress inflammation. Clinical trials failed to show efficacy of immunomodulatory therapies in sepsis $(36,37)$. Since bacterial sepsis shares some similarities with COVID-19, we may consider a new route for therapeutic intervention focused on mitochondrial cell transfer. Another option is therapy with engineered EVs containing mitochondria.

\section{RATIONALE FOR USING EXTRACELLULAR VESICLES IN COVID-19}

The immunomodulatory and regenerative potential of MSCs may be independent of direct cellular cross-talk (21, 38). MSCs act through a paracrine mechanism based primarily on EVs, which interact with neighboring target cells or can reach distant organs (39). Distinctions between the subtypes of EVs were previously based on subcellular origin, with exosomes being of endosomal origin and microvesicles derived from the cell membrane. However, given the historically contradictory definitions and inaccurate expectations of biogenesis associated with these terms, in 2018, ISEV recommended the use of new terms for EV subtypes that refer to their physical characteristics, such as size (small and medium/large EVs) or density; their biochemical composition (CD63+/CD81+- EVs, annexin A5stained EVs, etc.); or their cell of origin (MSC EVs, podocyte EVs, etc.) (40). EV biochemistry varies according to composition and cell source (41). EVs can carry membrane and cytosolic proteins, transcription factors, DNA, coding and non-coding RNAs and various signal transduction molecules (5, 21, 38, 42 ), acting on both physiological and pathological events, e.g., modulating the inflammatory response $(2,14)$. EVs also carry different cytokines and growth factors, such as interleukin (IL)- 6 and IL-10, transforming growth factor (TGF)- $\beta$, and hepatocyte growth factor (HGF) (43). In addition, EVs contain matrix-remodeling enzymes, such as matrix metalloproteinases (MMPs), heparanases, hyaluronidases, and tissue inhibitors of metalloproteinases (TIMPs); EV-mediated proteolytic activities have also been described, which might modulate the remodeling process and contribute to tissue repair (39). In this context, our group demonstrated that MSCs increased MMP-8 expression and decreased TIMP-1 expression in an experimental model of ARDS, suggesting an effect on the extracellular matrix (44). Rather than suppressing immune responses, EVs appear to act as true modulators, inducing regulatory responses and tolerance in order to restore homeostasis (45).

Administration of EVs has proven safe and effective in preclinical studies of lung injury and sepsis models (16, 18, 19, 46-48). In preclinical studies, EV therapy ameliorated acute lung injury $(49,50)$ and was equally or even more effective 
than MSCs in mitigating lung inflammation and pathological damage $(41,51)$. EVs have also been shown to attenuate $E$. coli and influenza infections $(47,48,52)$, including a mixed swine (H3N2, H1N1) and avian (H9N5, H7N2) influenzainduced lung injury model (48). The beneficial effects of EVs have further been observed in an ischemic stroke model (53, 54). Since COVID-19 may be associated with damage to other organs in addition to the lungs and has been associated with ischemic stroke, EVs could be a particularly promising therapy in this context (19). However, only one prospective study has evaluated the effects of EVs (specifically, exosomes from bone marrow-derived MSCs) in COVID-19 patients (55). Even though the inflammatory response was reduced significantly, and no adverse events were observed, this study encountered limitations regarding $\mathrm{EV}$ characterization and biological properties, the actual dose of EV administered, and how the injection of EVs was monitored. At the time of writing, there are three ongoing clinical trials of MSC-derived EVs for COVID-19 treatment, to be administered intravenously (ChiCTR2000030484) or by inhalation (NCT04276987, ChiCTR2000030261); however, recruitment has not yet begun.

It is important to consider both the source of MSCs from which EVs are derived, which can be obtained from different tissues and donors, and their preparation. Depending on these factors, MSCs and their EVs can have different therapeutic properties. Compared to bone marrow-derived MSCs, for instance, adipose tissue-derived MSCs express more tissue factor (an important initiator of coagulation in sepsis) and reduce hemocompatibility, which has been shown to vary according to donor and culture handling conditions $(56,57)$. Therefore, EVs obtained from adipose tissue-derived MSCs may have greater thrombogenic activity than those from bone marrowderived MSCs $(58,59)$, and thus should not be considered for use in COVID-19 patients $(5,19)$. Furthermore, the therapeutic effects of EVs are known to vary according to their preparation method, even when obtained from the same MSC source (53). Moreover, differences in donor parameters, including age, have been associated with significant variations in cytokine content, thus resulting in different effects on injury mitigation $(60,61)$.

In addition to their natural cargo, EVs can be loaded with biochemical compounds or genetically engineered to target infected cells, thus providing additional perspective for COVID19 treatment beyond MSC-derived EVs, including EV-based drug delivery, inhibition of EV biogenesis and uptake, and EVbased vaccines (41). The latter might be a particularly promising cell-free approach to COVID-19 treatment. Exosome vaccines may contain membrane-anchored ectodomains of SARS-CoV-2

\section{REFERENCES}

1. Liu S, Peng D, Qiu H, Yang K, Fu Z, Zou L. Mesenchymal stem cells as a potential therapy for COVID-19. Stem Cell Res Ther. (2020) 11:811. doi: 10.1186/s13287-020-01678-8

2. Basiri A, Pazhouhnia Z, Beheshtizadeh N, Hoseinpour M, Saghazadeh A. Regenerative medicine in COVID-19 treatment : components on their surface, facilitating cross-linking of the Bcell receptor (2). Exosome-based vaccines containing the spike (S) proteins of SARS-CoV-2, one of the structural proteins that mediate viral entry into the host cells (62), could induce high levels of neutralizing antibodies (63). In addition, the cargo of EV-based vaccines can be modified to include proteins and miRNAs to help modulate the immune response (62). However, further research is needed to assess the safety and clinical pharmacology of EV-based therapies in order to provide guidance for manufacturing, storage, dosing, and administration (19) before these potential treatments can be made more accessible worldwide $(14,17)$.

\section{CONCLUSION}

In the specific setting of COVID-19, administration of MSC-EVs may have several advantages over MSCs: (i) there is no risk of emboli formation in the injured microcirculation; (ii) no risk of mutagenicity or oncogenicity is observed; (iii) nebulized delivery can be used (despite several controversies regarding this route of administration); and (iv) tolerance of longer storage periods allow for later therapeutic use, reducing the stringency of storage and transportation requirements. In short, cell-free therapies should be considered a promising alternative for COVID-19 treatment. Clinical trials of EV-based therapies for COVID19 should clearly describe the dose, route of administration, characteristics of the administered EVs, timing of administration, any monitoring performed during administration, and detailed primary and secondary outcomes.

\section{AUTHOR CONTRIBUTIONS}

All authors conceived the concept of this article and wrote the manuscript.

\section{FUNDING}

This study was supported by the Brazilian Council for Scientific and Technological Development $(\mathrm{CNPq})$, the Rio de Janeiro State Research Foundation (FAPERJ), the National Institute of Science and Technology for Regenerative Medicine (INCT-REGENERA), Funding Authority for Studies and Projects (FINEP), and Inova Fiocruz/Oswaldo Cruz Foundation.

\section{ACKNOWLEDGMENTS}

The authors thank Filippe Vasconcellos, B.A., for his assistance in editing the manuscript.

3. Saleki K, Banazadeh M, Saghazadeh A, Rezaei N. The involvement of the central nervous system in patients with COVID-19. Rev Neurosci. (2020) 31:453-6. doi: 10.1515/revneuro-2020-0026

4. Robba C, Battaglini D, Pelosi P, Rocco PRM, Robba C, Battaglini D, et al. Multiple organ dysfunction in SARS-CoV-2 : MODS-CoV-2. Expert Rev 
Respir Med. (2020) 1-4. doi: 10.1080/17476348.2020.1778470. [Epub ahead of print].

5. Rogers CJ, Harman RJ, Bunnell BA, Schreiber MA, Xiang C, Wang FS, et al. Rationale for the clinical use of adipose-derived mesenchymal stem cells for COVID-19 patients. J Transl Med. (2020) 18:1-19. doi: 10.1186/s12967-020-02380-2

6. Chrzanowski W, Kim SY, McClements L. Can stem cells beat COVID-19: advancing stem cells and extracellular vesicles toward mainstream medicine for lung injuries associated with SARS-CoV-2 infections. Front Bioeng Biotechnol. (2020) 8:554. doi: 10.3389/fbioe.2020.00554

7. Battaglini D, Robba C, Ball L, Cruz FF, Silva PL, Pelosi P, et al. Emerging therapies for COVID-19 pneumonia. Expert Opin Investig Drugs. (2020) 1-5. doi: 10.1080/13543784.2020.1771694. [Epub ahead of print].

8. Russell CD, Millar JE, Baillie JK. Clinical evidence does not support corticosteroid treatment for 2019-nCoV lung injury. Lancet. (2020) 395:4735. doi: 10.1016/S0140-6736(20)30317-2

9. RECOVERY Collaborative Group, Horby P, Lim WS, Emberson JR, Mafham M, Bell JL, et al. Dexamethasone in hospitalized patients with Covid-19 - preliminary report. N Engl J Med. (2020). doi: 10.1056/NEJMoa2021436. [Epub ahead of print].

10. Golchin A, Seyedjafari E, Ardeshirylajimi A. Mesenchymal stem cell therapy for COVID-19: present or future. Stem Cell Rev Rep. (2020) 16:42733. doi: 10.1007/s12015-020-09973-W

11. Leng Z, Zhu R, Hou W, Feng Y, Yang Y, Han Q, et al. Transplantation of ACE2mesenchymal stem cells improves the outcome of patients with COVID-19 pneumonia. Aging Dis. (2020) 11:216. doi: 10.14336/AD.2020.0228

12. Wang J, Jiang M, Chen X, Montaner LJ. Cytokine storm and leukocyte changes in mild versus severe SARS-CoV-2 infection: review of 3939 COVID-19 patients in China and emerging pathogenesis and therapy concepts. J Leukoc Biol. (2020) 108:17-41. doi: 10.1002/JLB.3COVR0520-272R

13. Ulrich H, Pillat MM. CD147 as a target for COVID-19 treatment: suggested effects of azithromycin and stem cell engagement. Stem Cell Rev Rep. (2020) 16:434-40. doi: 10.1007/s12015-020-09976-7

14. Khoury M, Cuenca J, Cruz FF, Figueroa FE, Rocco PRM, Weiss DJ. Current status of cell-based therapies for respiratory virus infections: applicability to COVID-19. Eur Respir J. (2020) 55:2000858. doi: 10.1183/13993003.00858-2020

15. Cancio M, Ciccocioppo R, Rocco P, Levine B, Bronte V, Bollard CM, et al. Emerging trends in COVID-19 treatment: learning from inflammatory conditions associated with cellular therapies. Cytotherapy. (2020) 22:47481. doi: $10.1016 /$ j.jcyt.2020.04.100

16. Mahida RY, Matsumoto S, Matthay MA. Extracellular vesicles : a new frontier for research in acute respiratory. Am J Respir Cell Mol Biol. (2020) 63:1524. doi: $10.1165 / \mathrm{rcmb} .2019-0447 \mathrm{TR}$

17. Bari E, Ferrarotti I, Saracino L, Perteghella S, Torre ML, Corsico AG. Mesenchymal stromal cell secretome for severe COVID-19 infections: premises for the therapeutic use. Cells. (2020) 9:5-9. doi: 10.3390/cells9040924

18. Lopes-Pacheco M, Robba C, Rocco PRM, Pelosi P. Current understanding of the therapeutic benefits of mesenchymal stem cells in acute respiratory distress syndrome. Cell Biol Toxicol. (2020) 36:83-102. doi: 10.1007/s10565-019-09493-5

19. Borger V, Weiss DJ, Anderson JD, Bussolati B, Carter DRF, Dominici $\mathrm{M}$, et al. International Society for Extracellular Vesicles and International Society for Cell and Gene Therapy statement on extracellular vesicles from mesenchymal stromal cells and other cells: considerations for potential therapeutic agents to suppress coronavirus disease-19. Cytotherapy. (2020) 22:482-5. doi: 10.1016/j.jcyt.2020.05.002

20. Teixeira FG, Panchalingam KM, Assunção-Silva R, Serra SC, MendesPinheiro B, Patrício P, et al. Modulation of the mesenchymal Stem cell secretome using computer-controlled bioreactors: impact on neuronal cell proliferation, survival and differentiation. Sci Rep. (2016) 6:27791. doi: 10.1038/srep27791

21. Fatima F, Ekstrom K, Nazarenko I, Maugeri M, Valadi H, Hill AF, et al. Non-coding RNAs in mesenchymal stem cell-derived extracellular vesicles: deciphering regulatory roles in stem cell potency, inflammatory resolve, and tissue regeneration. Front Genet. (2017) 8:161. doi: 10.3389/fgene.2017.00161
22. Phelps J, Sanati-Nezhad A, Ungrin M, Duncan NA, Sen A. Bioprocessing of mesenchymal stem cells and their derivatives: toward cell-free therapeutics. Stem Cells Int. (2018) 2018:9415367. doi: 10.1155/2018/9415367

23. Lim SK, Giebel B, Weiss DJ, Witwer KW, Rohde E. Re: "Exosomes derived from bone marrow mesenchymal stem cells as treatment for severe COVID-19" by Sengupta et al. Stem Cells Dev. (2020) 29:8778. doi: $10.1089 / \mathrm{scd} .2020 .0089$

24. Maron-Gutierrez T, Laffey JG, Pelosi P, Rocco PRM. Cell-based therapies for the acute respiratory distress syndrome. Curr Opin Crit Care. (2014) 20:122-31. doi: 10.1097/MCC.0000000000000061

25. Islam MN, Das SR, Emin MT, Wei M, Sun L, Westphalen $K$, et al. Mitochondrial transfer from bone-marrow-derived stromal cells to pulmonary alveoli protects against acute lung injury. Nat Med. (2012) 18:759-65. doi: $10.1038 / \mathrm{nm} .2736$

26. Court AC, Le-Gatt A, Luz-Crawford P, Parra E, Aliaga-Tobar V, Bátiz $\mathrm{LF}$, et al. Mitochondrial transfer from MSCs to $\mathrm{T}$ cells induces treg differentiation and restricts inflammatory response. EMBO Rep. (2020) 21:e48052. doi: 10.15252/embr.201948052

27. Huang C, Wang Y, Li X, Ren L, Zhao J, Hu Y, et al. Clinical features of patients infected with 2019 novel coronavirus in Wuhan, China. Lancet. (2020) 395:497-506. doi: 10.1016/S0140-6736(20)30183-5

28. Harrell C, Fellabaum C, Jovicic N, Djonov V, Arsenijevic N, Volarevic V. Molecular mechanisms responsible for therapeutic potential of mesenchymal stem cell-derived secretome. Cells. (2019) 8:467. doi: 10.3390/ cells 8050467

29. Cheung MB, Sampayo-Escobar V, Green R, Moore ML, Mohapatra S, Mohapatra SS. Respiratory syncytial virus-infected mesenchymal stem cells regulate immunity via interferon beta and indoleamine-2,3-dioxygenase. PLoS ONE. (2016) 11:e0163709. doi: 10.1371/journal.pone.0163709

30. Picard M, McEwen BS, Epel ES, Sandi C. An energetic view of stress: focus on mitochondria. Front Neuroendocrinol. (2018) 49:7285. doi: 10.1016/j.yfrne.2018.01.001

31. Manoli I, Alesci S, Blackman MR, Su YA, Rennert OM, Chrousos GP. Mitochondria as key components of the stress response. Trends Endocrinol Metab. (2007) 18:190-8. doi: 10.1016/j.tem.2007.04.004

32. Singer M, Deutschman CS, Seymour C, Shankar-Hari M, Annane D, Bauer M, et al. The third international consensus definitions for sepsis and septic shock (sepsis-3). JAMA. (2016) 315:801-10. doi: 10.1001/jama.2016.0287

33. Heming N, Mazeraud A, Verdonk F, Bozza FA, Chrétien F, Sharshar T. Neuroanatomy of sepsis-associated encephalopathy. Crit Care. (2017) 21:65. doi: 10.1186/s13054-017-1643-z

34. Phinney DG, Di Giuseppe M, Njah J, Sala E, Shiva S, St Croix CM, et al. Mesenchymal stem cells use extracellular vesicles to outsource mitophagy and shuttle microRNAs. Nat Commun. (2015) 6:8472. doi: 10.1038/ ncomms 9472

35. Puhm F, Afonyushkin T, Resch U, Obermayer G, Rohde M, Penz T, et al. Mitochondria are a subset of extracellular vesicles released by activated monocytes and induce type I IFN and TNF responses in endothelial cells. Circ Res. (2019) 125:43-52. doi: 10.1161/CIRCRESAHA.118.314601

36. Sinha P, Matthay MA, Calfee CS. Is a "Cytokine storm" relevant to COVID-19? JAMA Intern Med. (2020) 6-8. doi: 10.1001/jamainternmed.2020.3313. [Epub ahead of print].

37. Singer M. The role of mitochondrial dysfunction in sepsis-induced multiorgan failure. Virulence. (2014) 5:66-72. doi: 10.4161/viru.26907

38. Fatima F, Nawaz M. Nexus between extracellular vesicles, immunomodulation and tissue remodeling: for good or for bad? Ann Transl Med. (2017) 5:139. doi: $10.21037 / \mathrm{atm} .2017 .03 .71$

39. Nawaz M, Shah N, Zanetti B, Maugeri M, Silvestre R, Fatima F, et al. Extracellular vesicles and matrix remodeling enzymes: the emerging roles in extracellular matrix remodeling, progression of diseases and tissue repair. Cells. (2018) 7:167. doi: 10.3390/cells7100167

40. Théry C, Witwer KW, Aikawa E, Alcaraz MJ, Anderson JD, Andriantsitohaina $\mathrm{R}$, et al. Minimal information for studies of extracellular vesicles 2018 (MISEV2018): a position statement of the International Society for Extracellular Vesicles and update of the MISEV2014 guidelines. J Extracell Vesicles. (2019) 8:1535750. doi: 10.1080/20013078.2018.1535750 
41. Hassanpour M, Rezaie J, Nouri M, Panahi Y. The role of extracellular vesicles in COVID-19 virus infection. Infect Genet Evol. (2020) 85:104422. doi: 10.1016/j.meegid.2020.104422

42. Joo HS, Suh JH, Lee HJ, Bang ES, Lee JM. Current knowledge and future perspectives on mesenchymal stem cell-derived exosomes as a new therapeutic agent. Int J Mol Sci. (2020) 21:727. doi: 10.3390/ijms21030727

43. Qiu G, Zheng G, Ge M, Wang J, Huang R, Shu Q. Functional proteins of mesenchymal stem cell-derived extracellular vesicles. Stem Cell Res Ther. (2019) 10:359. doi: 10.1186/s13287-019-1484-6

44. Maron-Gutierrez T, Silva JD, Asensi KD, Bakker-Abreu I, Shan Y, Diaz BL, et al. Effects of mesenchymal stem cell therapy on the time course of pulmonary remodeling depend on the etiology of lung injury in mice. Crit Care Med. (2013) 41:319-33. doi: 10.1097/CCM.0b013e31828a663e

45. Giebel B, Hermann DM. Identification of the right cell sources for the production of therapeutically active extracellular vesicles in ischemic stroke. Ann Transl Med. (2019) 7:188. doi: 10.21037/atm.2019.03.49

46. Zhu YG, Feng XM, Abbott J, Fang XH, Hao Q, Monsel A, et al. Human mesenchymal stem cell microvesicles for treatment of Escherichia coli endotoxin-induced acute lung injury in mice. Stem Cells. (2014) 32:11625. doi: 10.1002/stem.1504

47. Monsel A, Zhu YG, Gennai S, Hao Q, Hu S, Rouby JJ, et al. Therapeutic effects of human mesenchymal stem cell-derived microvesicles in severe pneumonia in mice. Am J Respir Crit Care Med. (2015) 192:32436. doi: 10.1164/rccm.201410-1765OC

48. Khatri M, Richardson LA, Meulia T. Mesenchymal stem cell-derived extracellular vesicles attenuate influenza virus-induced acute lung injury in a pig model. Stem Cell Res Ther. (2018) 9:17. doi: 10.1186/s13287-018-0774-8

49. Shah TG, Predescu D, Predescu S. Mesenchymal stem cells-derived extracellular vesicles in acute respiratory distress syndrome: a review of current literature and potential future treatment options. Clin Transl Med. (2019) 8:25. doi: 10.1186/s40169-019-0242-9

50. Wu X, Liu Z, Hu L, Gu W, Zhu L. Exosomes derived from endothelial progenitor cells ameliorate acute lung injury by transferring miR-126. Exp Cell Res. (2018) 370:13-23. doi: 10.1016/j.yexcr.2018.06.003

51. Abreu SC, Weiss DJ, Rocco PRM. Extracellular vesicles derived from mesenchymal stromal cells: a therapeutic option in respiratory diseases? Stem Cell Res Ther. (2016) 7:53. doi: 10.1186/s13287-016-0317-0

52. Hao Q, Gudapati V, Monsel A, Park JH, Hu S, Kato H, et al. Mesenchymal stem cell-derived extracellular vesicles decrease lung injury in mice. J Immunol. (2019) 203:1961-72. doi: 10.4049/jimmunol.18 01534

53. Wang C, Börger V, Sardari M, Murke F, Skuljec J, Pul R, et al. Mesenchymal stromal cell-derived small extracellular vesicles induce ischemic neuroprotection by modulating leukocytes and specifically neutrophils. Stroke. (2020) 51:1825-34. doi: 10.1161/STROKEAHA.119.0 28012
54. Doeppner TR, Herz J, Görgens A, Schlechter J, Ludwig A-K, Radtke S, et al. Extracellular vesicles improve post-stroke neuroregeneration and prevent postischemic immunosuppression. Stem Cells Transl Med. (2015) 4:113143. doi: $10.5966 / \mathrm{sctm} .2015-0078$

55. Sengupta V, Sengupta S, Lazo A, Woods P, Nolan A, Bremer N. Exosomes derived from bone marrow mesenchymal stem cells as treatment for severe COVID-19. Stem Cells Dev. (2020) 29:747-54. doi: 10.1089/scd. 2020.0080

56. George MJ, Prabhakara K, Toledano-Furman NE, Wang YW, Gill BS, Wade $\mathrm{CE}$, et al. Clinical cellular therapeutics accelerate clot formation. Stem Cells Transl Med. (2018) 7:731-9. doi: 10.1002/sctm.18-0015

57. Moll G, Rasmusson-Duprez I, Von Bahr L, Connolly-Andersen AM, Elgue G, Funke L, et al. Are therapeutic human mesenchymal stromal cells compatible with human blood? Stem Cells. (2012) 30:1565-74. doi: 10.1002/stem.1111

58. Chance TC, Rathbone CR, Kamucheka RM, Peltier GC, Cap AP, Bynum JA. The effects of cell type and culture condition on the procoagulant activity of human mesenchymal stromal cell-derived extracellular vesicles. J Trauma Acute Care Surg. (2019) 87:S74-82. doi: 10.1097/TA.0000000000002225

59. Silachev D, Goryunov K, Shpilyuk M, Beznoschenko O, Morozova N, Kraevaya E, et al. Effect of MSCs and MSC-derived extracellular vesicles on human blood coagulation. Cells. (2019) 8:258. doi: 10.3390/cells8030258

60. Huang R, Qin C, Wang J, Hu Y, Zheng G, Qiu G, et al. Differential effects of extracellular vesicles from aging and young mesenchymal stem cells in acute lung injury. Aging. (2019) 11:7996-8014. doi: 10.18632/aging.102314

61. Kordelas L, Rebmann V, Ludwig AK, Radtke S, Ruesing J, Doeppner TR, et al. MSC-derived exosomes: a novel tool to treat therapy-refractory graft-versushost disease. Leukemia. (2014) 28:970-3. doi: 10.1038/leu.2014.41

62. Wang YD, Li Y, Xu G Bin, Dong XY, Yang XA, Feng ZR, et al. Detection of antibodies against SARS-CoV in serum from SARS-infected donors with ELISA and western blot. Clin Immunol. (2004) 113:14550. doi: 10.1016/j.clim.2004.07.003

63. Kuate S, Cinatl J, Doerr HW, Überla K. Exosomal vaccines containing the S protein of the SARS coronavirus induce high levels of neutralizing antibodies. Virology. (2007) 362:26-37. doi: 10.1016/j.virol.2006.12.011

Conflict of Interest: The authors declare that the research was conducted in the absence of any commercial or financial relationships that could be construed as a potential conflict of interest.

Copyright (C) 2020 Maron-Gutierrez and Rocco. This is an open-access article distributed under the terms of the Creative Commons Attribution License (CC BY). The use, distribution or reproduction in other forums is permitted, provided the original author(s) and the copyright owner(s) are credited and that the original publication in this journal is cited, in accordance with accepted academic practice. No use, distribution or reproduction is permitted which does not comply with these terms. 